\title{
An Arabidopsis Zinc Finger Protein Increases Abiotic Stress Tolerance by Regulating Sodium and Potassium Homeostasis, Reactive Oxygen Species Scavenging and Osmotic Potential
}

\author{
Dandan Zang ${ }^{1}$, Hongyan Li $^{1}$, Hongyun X ${ }^{1}{ }^{1}$, Wenhui Zhang ${ }^{1}$, Yiming Zhang ${ }^{1}$, Xinxin Shi ${ }^{1}$ \\ and Yucheng Wang ${ }^{1,2 *}$
}

\begin{abstract}
${ }^{1}$ State Key Laboratory of Forest Genetics and Tree Breeding, Northeast Forestry University, Harbin, China, ${ }^{2}$ Key Laboratory of Biogeography and Bioresource in Arid Land, Xinjiang Institute of Ecology and Geography, Chinese Academy of Sciences, Xinjiang, China
\end{abstract}

OPEN ACCESS

Edited by:

Mohammad Anwar Hossain, Bangladesh Agricultural University,

Bangladesh

Reviewed by:

Xiping Wang,

Northwest A\&F University, China

Tzvetina Brumbarova,

University of Düsseldorf, Germany

*Correspondence: Yucheng Wang wangyucheng@ms.xjb.ac.cn

Specialty section: This article was submitted to

Plant Biotechnology,

a section of the journal

Frontiers in Plant Science

Received: 16 June 2016 Accepted: 10 August 2016 Published: 24 August 2016

Citation:

Zang $D, L i H, X u H$, Zhang $W$, Zhang Y, Shi X and Wang Y (2016) An Arabidopsis Zinc Finger Protein Increases Abiotic Stress Tolerance by

Regulating Sodium and Potassium Homeostasis, Reactive Oxygen Species Scavenging and Osmotic Potential. Front. Plant Sci. 7:1272. doi: 10.3389/fpls.2016.01272
Plant zinc finger proteins (ZFPs) comprise a large protein family and they are mainly involved in abiotic stress tolerance. Although Arabidopsis RING/FYVE/PHD ZFP At5g62460 (AtRZFP) is found to bind to zinc, whether it is involved in abiotic stress tolerance is still unknown. In the present study, we characterized the roles of AtRZFP in response to abiotic stresses. The expression of AtRZFP was induced significantly by salt and osmotic stress. AtRZFP positively mediates tolerance to salt and osmotic stress. Additionally, compared with wild-type Arabidopsis plants, plants overexpressing AtRZFP showed reduced reactive oxygen species (ROSs) accumulation, enhanced superoxide dismutase and peroxidase activity, increased soluble sugars and proline contents, reduced $\mathrm{K}^{+}$loss, decreased $\mathrm{Na}^{+}$accumulation, stomatal aperture and the water loss rate. Conversely, AtRZFP knockout plants displayed the opposite physiological changes when exposed to salt or osmotic stress conditions. These data suggested that AtRZFP enhances salt and osmotic tolerance through a series of physiological processes, including enhanced ROSs scavenging, maintaining $\mathrm{Na}^{+}$and $\mathrm{K}^{+}$homeostasis, controlling the stomatal aperture to reduce the water loss rate, and accumulating soluble sugars and proline to adjust the osmotic potential.

Keywords: Arabidopsis thaliana, AtRZFP, abiotic stress, zinc finger proteins, ROS scavenging analysis, $\mathrm{Na}^{+}$and $\mathrm{K}^{+}$content

\section{INTRODUCTION}

Zinc-finger proteins (ZFPs) are known to play various important roles in diverse organisms (Luo et al., 2012a), and their expression responds to various abiotic stresses (Sun et al., 2010). According to the number and order of the $\mathrm{C}$ and $\mathrm{H}$ residues in the secondary structure of the finger, ZFPs are classified into nine groups: $\mathrm{C} 2 \mathrm{H} 2, \mathrm{C} 8, \mathrm{C} 6, \mathrm{C} 3 \mathrm{HC} 4, \mathrm{C} 2 \mathrm{HC}, \mathrm{C} 2 \mathrm{HC} 5, \mathrm{C} 4, \mathrm{C} 4 \mathrm{HC} 3$, and $\mathrm{C} 3 \mathrm{H}$ (Schumann et al., 2007; Gao et al., 2012; Gupta et al., 2012). Based on their structural diversities, 
ZFPs are categorized into 23 subfamilies, such as GATA, FYVE, TDDP, RBPO, A20, TFIIB, Dof, CDGSH, PADPP, LYAR, ZK, TAZ, MSRING, Ubox, Bbox, NFX1, AN, UBR, Ring, WRKY, DHHC, PHD, and CHY (Li W.T. et al., 2014).

Zinc finger proteins are a large family in plant kingdoms; for instance, there are 176 ZFPs in Arabidopsis thaliana and 126 ZFPs in wheat. Although ZFPs are abundant in plants, only a handful of them have been characterized functionally. These characterized ZFPs are involved in plant development (Li X.J. et al., 2014; Seok et al., 2016), regulation of plant height (Liu et al., 2011; Sendon et al., 2014), root development (Liu et al., 2013), flower development (Yang et al., 2014), seed germination (Baek et al., 2015), secondary wall thickening and anther development (Chai et al., 2015), and fruit ripening (Weng et al., 2015). ZFP family proteins are involved in resistance to biotic stresses, such as rice blast fungus infection ( $\mathrm{Li} \mathrm{Y}$. et al., 2014; Cao et al., 2016). In addition, ZFPs play important roles in abiotic stress. Cheuk and Houde (2016) investigated 53 Q-type $\mathrm{C} 2 \mathrm{H} 2$ zinc finger protein (TaZFPs) from Triticum aestivum, and showed that these TaZFPs are mainly responsive to high light (44/53), $\mathrm{H}_{2} \mathrm{O}_{2}$ (37/53), drought (37/53), and flooding $(31 / 53) ; 16$ genes were responsive to all stresses tested. This result indicated that these TaZFPs play important roles in abiotic stress resistance. A study of $109 \mathrm{C} 2 \mathrm{H} 2$ zinc-finger (C2H2-ZF) proteins from Populus trichocarpa showed that most of them contain phytohormone or abiotic stress-related cis-elements in their promoter regions. Quantitative real-time RT-PCR analysis suggested that these $\mathrm{C} 2 \mathrm{H} 2-\mathrm{ZF}$ genes are involved significantly in salt, drought, and heat responses (Liu et al., 2015). Abiotic stress alters the metabolic balance to induce the generation of reactive oxygen species (ROS; Miller et al., 2008). Excess ROS causes oxidative damage to macromolecules, including DNA, proteins and lipids. Damage to macromolecules will lead to modulation of gene expression, the cell cycle, cell metabolism, cell adhesion, and cell death, ultimately interfering with their normal function (Kadir et al., 2013). Therefore, control of the ROS level is critical for abiotic tolerance. ZFPs plays important roles in ROS scavenging resulting from abiotic stress. ZFPs regulate the expression of a series of stress-activated genes in plants via ROS signaling to resist salt, drought or oxidative stress. Genes involved in antioxidation are regulated significantly by ZFPs, which leads to elevated antioxidant enzyme activities and reduced ROS accumulation; thus, enhancing abiotic stress tolerance (Davletova et al., 2005; Sun et al., 2010; Zhang et al., 2014; Baek et al., 2015; Fan et al., 2015). In addition to ROS pathways, other physiological adaptations to abiotic stress tolerance mediated by ZFPs have been studied. ZFPs can confer abiotic stress tolerance by increasing the contents of abscisic acid (ABA), proline, soluble sugars or chlorophyll, and reducing the water loss rate (Luo et al., 2012b; Wang et al., 2016). ZFPs are also involved in gibberellins (GA) signaling in the regulation of the stress response. Transgenic plants overexpressing the ZFPs OsDOG (Liu et al., 2011) and ZFP185 (Zhang Y. et al., 2016) displayed a reduced GA content, dwarf phenotypes and sensitivity to abiotic stress. However, plants overexpressing BBX24 (Yang et al., 2014) also showed reduced GA content, but plants expressing BBX24 showed normal growth phenotype and enhanced salt and drought stress tolerance (Yang et al., 2014). ZFPS are also involved in high temperature (Kim et al., 2015) and $\mathrm{H}^{+}$ tolerance (Fan et al., 2015). Although many ZFPs regulate abiotic stress tolerance positively, some ZFPs regulate abiotic stress tolerance negatively. For example, arginine-rich tandem zincfinger proteins (RR-TZF) AtTZF3 regulate seed germination negatively under salt stress conditions (D'Orso et al., 2015). Soybean GmZFP3 might be involved in the ABA-dependent signaling pathway, and plays a negative role in drought tolerance (Zhang D. et al., 2016). Analysis of ABA-related marker gene expression in Arabidopsis suggested that GmZFP3 might be involved in the ABA-dependent pathway during the drought stress response. OsDOG and ZFP185 also play negative roles in abiotic stress tolerance (Liu et al., 2011; Zhang Y. et al., 2016).

Additionally, ZFPs also interact with different proteins to mediate abiotic stress tolerance. For instance, Bogamuwa and Jang (2016) showed that Tandem CCCH Zinc Finger protein (ZFP), AtTZF could interact with the proteins such as Mediator of ABA-Regulated Dormancy 1 (MARD1) and Responsive to Dehydration 21A (RD21A) to perform its functions in ABA, GA, and phytochrome-mediated seed germination responses. Zinc Finger of $A$. thaliana12 (ZAT12) is induced by abiotic stress and interacts with FER-LIKE IRON DEFICIENCYINDUCED TRANSCRIPTION FACTOR (FIT). Under Fe deficient conditions, $\mathrm{H}_{2} \mathrm{O}_{2}$ levels were increased in a FITdependent manner, and the FIT protein was stabilized by $\mathrm{H}_{2} \mathrm{O}_{2}$ in the presence of ZAT12. ZAT12 has a negative role in $\mathrm{Fe}$ acquisition mediated by $\mathrm{H}_{2} \mathrm{O}_{2}$ signaling in the $\mathrm{Fe}$ deficiency responses (Le et al., 2016). ZAT6 interacts with, and is phosphorylated by, a stress-responsive mitogen-activated protein kinase, MPK6. Phosphorylation of ZAT6 by MPK6 is necessary for ZAT6's role in seed germination under salt and osmotic stress (Liu et al., 2013).

Previously, to identify the RING/FYVE/PHD ZFP involved in abiotic stress, we acquired some RING/FYVE/PHD ZFPs T-DNA insertion mutants from the Arabidopsis Biological Resource Centre (ABRC). We use DAB and Evans blue staining of these mutants to screen them initially for those that were sensitive to salt and mannitol stress. According to DAB and Evans blue staining, At5g62460 mutant plants (SALK_119330) was sensitive to salt stress compared with WT plants, implying that At5g62460 (AtRZFP) might be involved in the abiotic stress response, and was selected for further study. Our study showed that AtRZFP could increase tolerance to salt and osmotic stress, and we further revealed the physiological changes modulated by AtRZFP in response to abiotic stress. Our results provide useful insights into the function of ATRZFP in the regulation of salt and osmotic stress tolerance in A. thaliana.

\section{MATERIALS AND METHODS}

\section{Plant Materials, Growth Conditions, and Treatments}

A. thaliana Columbia type $(\mathrm{Col})$ plants were used in this study. The T-DNA insertion At5g62460 mutant (SALK_119330) was 
obtained from the ABRC. Seeds were surface sterilized and seeded on $1 / 2 \mathrm{MS}$ solid medium containing $2 \%$ sucrose at $22^{\circ} \mathrm{C}$ under a $16 \mathrm{~h}$ light $/ 8 \mathrm{~h}$ dark photoperiod. To analyze the expression of AtRZFP in response to abiotic stress, 4-week-old Arabidopsis plants were watered with a solution of $150 \mathrm{mM} \mathrm{NaCl}$ or $200 \mathrm{mM}$ mannitol on their roots. After treatment for 3, 6, 12, and $24 \mathrm{~h}$, the roots and aerial parts of plants were harvested for expression analysis. Plants watered with fresh water were harvested at corresponding time points as controls.

\section{Plasmid Constructs and Plant Transformation}

The coding sequence (CDS) of ZFP (AtRZFP) was cloned into the pROK2 vector (Hilder et al., 1987) under the control of CaMV 35S promoter to generate the 35S:ZFP construct, and was transformed into Arabidopsis plants using the flower-dipping method. Six transgenic lines were obtained (OE lines). The diagram of 35S:AtRZFP construct used for transformation is shown in Figure 2A. The AtRZFP knockout lines (SALK_119330) were homozygous for two generations, and the diagram of the T-DNA insertion positions in the SALK_119330 mutant plant is shown in Figure 2C, which represent a single allele of At5g62460. The expression of AtRZFP in (OE) lines and the SALK_119330 individual plants was monitored using quantitative real-time reverse transcription PCR (qRT-PCR) analysis.

\section{Subcellular Location and Western Blotting Analysis}

The CDS of AtRZFP, without its stop codon, was ligated in-frame to the $\mathrm{N}$-terminus of the green fluorescent protein (GFP) under the control of CaMV 35S promoter (35S:ZFPGFP). GFP under control of $35 \mathrm{~S}$ promoter was also generated (35S:GFP). All primers used to make these constructs are shown in Supporting Information Supplementary Table S1. For subcellular location analysis, the constructs 35S:AtRZFPGFP and 35S:GFP were introduced separately into onion epidermal cells using particle bombardment (Bio-Rad, Hercules, CA, USA). The transformed cells were analyzed under an LSM700 confocal laser microscope (Zeiss, Jena, Germany). GUS activity analysis was performed (Jefferson et al., 1987). For western blotting analysis, proteins were isolated from onion epidermal cells expressing 35S:AtRZFP-GFP transformed via particle bombardment or non-transformed onion epidermal cells (control). Western blot was performed according to the procedures described by Han et al. (2005), and an antiGFP antibody (Beyotime, Shanghai, China) was used to detect 35S:AtRZFP-GFP.

\section{AtRZFP Expression Assays in Response to Treatments}

Arabidopsis seedlings were grown in a greenhouse under normal conditions. AtRZFP gene expression in response to various treatments was analyzed. Roots and leaves of 4-weeks-old seedlings were treated with $150 \mathrm{mM} \mathrm{NaCl}$ or $200 \mathrm{mM}$ mannitol. Roots and leaves were sampled at 3, 6, 12, and $24 \mathrm{~h}$ after each treatment, and well watered plants were harvested at the corresponding time points as controls for RNA extraction.

\section{Stress Tolerance Assays}

$\mathrm{T}_{3}$ generation lines of the AtRZFP transformed Arabidopsis were randomly selected (OE 3 and OE 4) for stress tolerance. For the germination rate assay, the seeds were sown on half-strength Murashige-Skoog (1/2 MS) medium (as control), or 1/2 MS supplied with $150 \mathrm{mM} \mathrm{NaCl}$ or $200 \mathrm{mM}$ mannitol. After 1 week, the germination rates of the OE transgenic lines, wild-type (WT) and two SALK_119330 individual plants (KO 4 and KO 5) were calculated. For the stress tolerance assay, seeds were sown on $1 / 2$ MS medium for 3 days. After germination, the seeds were transferred into $1 / 2 \mathrm{MS}$ medium (as the control condition) or $1 / 2$ MS medium supplied with $150 \mathrm{mM} \mathrm{NaCl}$ or $200 \mathrm{mM}$ mannitol. After 2 weeks, the root length and fresh weights (FWs) were measured. The experiments were conducted with three independent biological replications.

\section{qRT-PCR Analysis}

Total RNA was isolated from Arabidopsis using the Trizol reagent (Promega, Madison, WI, USA), reversely transcribed into cDNA using the Primescript ${ }^{\mathrm{TM}} \mathrm{RT}$ reagent kit (Takara), and diluted with ultra-pure water (MilliQ) to $100 \mu \mathrm{l}$ as the PCR template. The tubulin $\beta$-2 (Locus number AT5G62690) and Actin 7 (Locus number AT5G09810) genes were used as internal references. All primers used are shown in Supplementary Table S2. qRT-PCR was carried out on an Opticon 2 System (Bio-Rad). The PCR reaction mixture contained $10 \mu \mathrm{l}$ of SYBR Green Real-time PCR Master Mix (Toyobo), $0.5 \mu \mathrm{M}$ of each forward and reverse primers, and $2 \mu \mathrm{l}$ of cDNA template (equivalent to $20 \mathrm{ng}$ of total RNA) in a reaction volume of $20 \mu \mathrm{l}$. The PCR conditions were as follows: $94^{\circ} \mathrm{C}$ for $30 \mathrm{~s}$; followed by 45 cycles at $94^{\circ} \mathrm{C}$ for $12 \mathrm{~s}$, $60^{\circ} \mathrm{C}$ for $30 \mathrm{~s}, 72^{\circ} \mathrm{C}$ for $40 \mathrm{~s}$; and $1 \mathrm{~s}$ at $82^{\circ} \mathrm{C}$ for plate reading. A melting curve of each sample was generated to evaluate the quality of the amplified product. Three biological replicates were conducted, and the expression levels were calculated using the $2^{-\Delta \Delta C t}$ method.

\section{Detection of ROS Accumulation and Cell Death using Biochemical Staining}

Detached leaves from different Arabidopsis lines were treated with $200 \mathrm{mM} \mathrm{NaCl}$ or $300 \mathrm{mM}$ mannitol for 0,6 , and $12 \mathrm{~h}$, and then used for histochemical staining analysis. The leaves were infiltrated with DAB or NBT, which allowed the detection of $\mathrm{H}_{2} \mathrm{O}_{2}$ and $\mathrm{O}_{2}{ }^{-}$, respectively, according to the method (Fryer et al., 2002). Evans Blue staining was performed to investigate the cell death, as described (Zhang L. et al., 2011). $\mathrm{H}_{2} \mathrm{O}_{2}$ was measured following the protocol of Thordal-Christensen et al. (1997).

\section{Analysis of Physiological Changes Involved in Abiotic Stress Tolerance}

For the physiological studies, OE, WT, and two SALK_119330 individual plants were treated with $150 \mathrm{mM} \mathrm{NaCl}$ or $200 \mathrm{mM}$ mannitol for 5 days and harvested for analysis. Measurement of the activities of SOD and POD were conducted according to the description (Dong and Chin, 2000). The MDA content was determined (Dhindsa et al., 1981). Electrolyte leakage was 
measured following the protocol (Sreenivasulu et al., 2000). Proline content was measured (Bates et al., 1973).

To measure the soluble carbohydrate content, $0.5 \mathrm{~g}$ of sample was ground into a fine powder, and was added with $5 \mathrm{ml}$ of $80 \%$ ethanol at $80^{\circ} \mathrm{C}$ for $30 \mathrm{~min}$. The extracting solution was then centrifuged at $12000 \mathrm{rpm}$ for $10 \mathrm{~min}$. After centrifugation, the supernatant was diluted with $80 \%$ ethanol to $10 \mathrm{~mL}$. One $\mathrm{mL}$ of the mixture and $5 \mathrm{~mL}$ anthrone were heated in boiling water for $15 \mathrm{~min}$, cooled immediately and placed in the dark for $30 \mathrm{~min}$. The absorbance of the supernatant was detected at $620 \mathrm{~nm}$, with water as the background. One sample per line was quantified three times.

To determine the $\mathrm{Na}^{+}$and $\mathrm{K}^{+}$contents, 1 week-old Arabidopsis seedlings were grown in a greenhouse supplemented without or with $150 \mathrm{mM} \mathrm{NaCl}$. The leaves and roots were then dried in an oven at $80^{\circ} \mathrm{C}$ for $48 \mathrm{~h}$ for weighting. The dried sample $(0.1 \mathrm{~g})$ was added with $10 \mathrm{~mL}$ deionized water, heated in boiling water for $2 \mathrm{~h}$, cooled immediately and centrifuged at $12000 \mathrm{rpm}$ for $15 \mathrm{~min}$. After centrifugation, the supernatant was used for cation content determination and water was used as the background in an atomic absorption spectrophotometer, as described previously (Chen et al., 2005; Ma et al., 2011).

To assay the water loss rate, the detached leaves from each line were weighed immediately as the FW, and incubated on a clean bench at a relative humidity of about $50 \%$, and their weights were measured at designated time intervals as the desiccated weight. After measurement, leaves were oven-dried at $80^{\circ} \mathrm{C}$ to a constant dry weight (DW). Water loss rates (\%) were calculated according to the formula: Water loss rate $(\%)=1$-(desiccated weight-DW $) /($ FW-DW $) \times 100$.

Stomatal apertures were measured according to Pei et al. (1997). Epidermal strips from leaves of different lines were floated in a solution containing $0 \mathrm{mM}$ (control) or $150 \mathrm{mM} \mathrm{NaCl}, 10 \mathrm{mM}$ Mes- $\mathrm{KOH}$, and $\mathrm{pH} 6.15$ for $3 \mathrm{~h}$ under light $\left(150 \mu \mathrm{mol} \cdot \mathrm{m}^{-2} \cdot \mathrm{s}^{-1}\right)$ at room temperature, after which they were observed under a light microscope (Olympus BX43, Japan). Leaves similar in size were taken from different lines at the same time. Three leaves were selected from each line or plant, 3 different epidermis parts were observed in each leaf, and 10 stomata guard cells in each epidermis part were measured. The length and width of the stomatal apertures were determined using the software Image $\mathrm{J}^{1}$. The ratios of width to length were then calculated.

\section{CoroNa-Green Staining}

To visualize the $\mathrm{Na}^{+}$distributions in the root cells of Col0, OE lines and two SALK_119330 individual plants, the intracellular $\mathrm{Na}^{+}$specific fluorescent indicator CoroNa-Green AM (Invitrogen Corp, Carlsbad, CA, USA) was used. Five-dayold seedlings grown on 1/2 MS medium were transferred to fresh medium containing $0 \mathrm{mM}$ (control) or $150 \mathrm{mM} \mathrm{NaCl}$ for $72 \mathrm{~h}$. The seedlings were then washed 2-3 times with distilled water and stained with $10 \mu \mathrm{M}$ CoroNa-Green AM in the presence of pluronic acid (0.02\%, Invitrogen) for $3 \mathrm{~h}$. The incubated sections were visualized under an LSM700 microscope (Zeiss, Jena, Germany) at excitation and emission wavelengths of 488

${ }^{1}$ http://rsbweb.nih.gov/ij and $516 \mathrm{~nm}$, respectively, as described by Oh et al. (2010). The experiments were conducted with three independent biological replications.

\section{Statistical Analyses}

Statistical analyses were conducted using the SPSS 16.0 software package (SPSS Inc, Chicago, IL, USA). Data were compared using one-way ANOVA and differences were considered statistically significant $\mathrm{d}$ at $P<0.05$.

\section{RESULTS}

\section{The Expression of AtRZFP Is Induced by $\mathrm{NaCl}$ and Mannitol}

To study the expression of AtRZFP in response to $\mathrm{NaCl}$ and mannitol stress, real-time RT-PCR was carried out. In leaves, the expression of AtRZFP was induced greatly by $\mathrm{NaCl}$ or mannitol stress for 3 and $6 \mathrm{~h}$, but decreased as stress continued for 12 and $24 \mathrm{~h}$ (Figures 1A,C). Interestingly, AtRZFP showed similar expression patterns in leaves when exposed to $\mathrm{NaCl}$ or mannitol stress (Figures 1A,C). The expression of AtRZFP was highly induced by $\mathrm{NaCl}$ in roots under $\mathrm{NaCl}$ stress for 3 to $24 \mathrm{~h}$, and reached a peak at $24 \mathrm{~h}$ (Figure 1B). In roots, the expression of AtRZFP was highly induced when exposed to mannitol stress for $3 \mathrm{~h}$, but decreased thereafter (Figure 1D). These results showed that the expression of AtRZFP responded to salt and osmotic stress, suggesting that AtRZFP plays a role the abiotic stress response.

\section{AtRZFP Is Located to Cytoplasm and Membrane}

To investigate the subcellular location of AtRZFP, the construct of 35S:AtRZFP-GFP was produced and transformed into onion epidermal cells. The green fluorescent signal from AtRZFP-GFP was observed clearly in the cytoplasm and membrane, while the 35S:GFP control was distributed uniformly throughout the cells (Figures 1E-J). Meanwhile, western blotting showed that the AtRZFP-GFP protein had been successfully expressed with no degradation (Figure $\mathbf{1 K}$ ). These results demonstrated that AtRZFP localizes to the cytoplasm and membrane.

\section{Generation of AtRZFP Overexpressing or Knockout Plants}

The results showed that the expression level of AtRZFP was significantly elevated in all $\mathrm{OE}$ transgenic lines, and two independent $\mathrm{T}_{3}$ homozygous lines (OE 3 and $\mathrm{OE} 4$ lines) with highest (increased by 256-fold) and middle (increased by 128 -fold) expression levels, respectively, were selected for subsequent studies (Figure 2B). Seven SALK_119330 individual plants (termed KO 1-7) were obtained, and the expression of AtRZFP was analyzed in these plants. The results showed that the expression of AtRZFP in all these seven individual plants was downregulated, and two individual plants, KO 4 and KO 5, which had the lowest expression levels, were selected for subsequent studies (Figure 2D). 


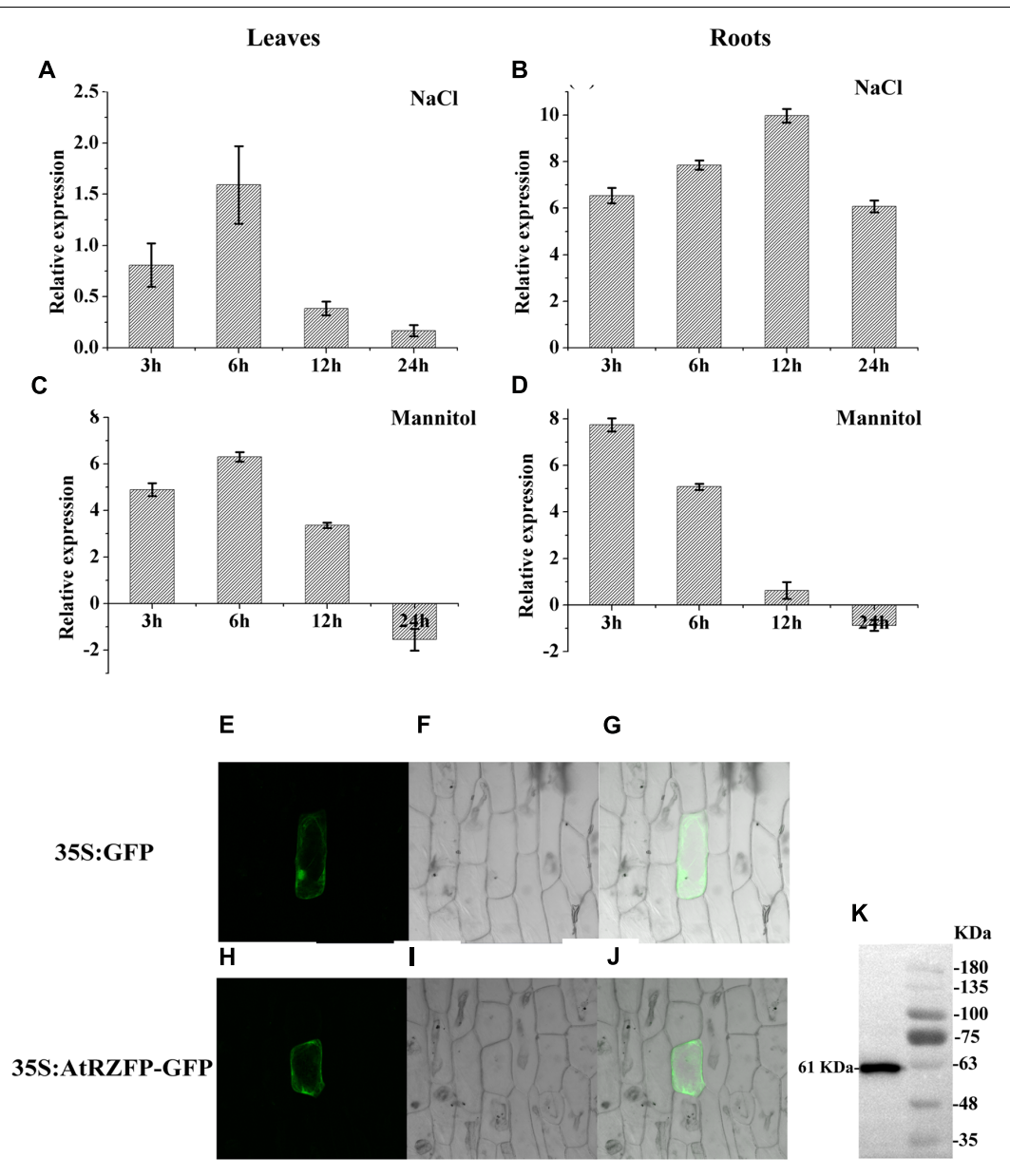

FIGURE 1 | Expression and subcellular localization of AtRZFP. (A-D) Well watered plants were harvested at each stress time point as controls. The expression of AtRZFP at each treatment time point was normalized by the expression of the AtRZFP transcript in the control harvested at the corresponding time. The expression data were log2 transformed. (E-J) The fusion construct for 35S:AtRZFP-GFP and the 35S:GFP control vector were introduced into onion epidermal cells by particle bombardment. (E,H) GFP fluorescence; (F,I) onion peel cells imaged under bright field illumination; (G,J) the merged images of bright-field and GFP; (K) Western blotting analysis of the expression of the AtRZFP-GFP protein. The full-length AtRZFP-GFP protein with the expected size of 61 kDa was detected.

\section{AtRZFP Increases Salt and Osmotic Stress Tolerance}

To characterize the function of AtRZFP in abiotic stress tolerance, three types of plants with different AtRZFP expression levels were studied, i.e., the WT plants, two independent $\mathrm{T}_{3}$ homozygous lines overexpressing AtRZFP, OE 3 and 4, and two AtRZFP mutant lines (KO 4 and $\mathrm{KO} 5$ ). Under normal growth conditions, no difference in seed germination rates, growth phenotype, FWs and root lengths were observed among the studied lines (Figure 3), indicating that overexpression or knockout of AtRZFP in Arabidopsis plants does not affect seed germination, growth rate and phenotype. However, after treatment with $\mathrm{NaCl}$ or mannitol, the two OE lines had significantly higher seed germination rates, FWs and root lengths compared with the WT plants; whereas both KO 4 and KO 5 had lower of seed germination rates, FWs and root lengths compared with the WT plants (Figure 3). These results indicated that overexpression of AtRZFP could increase tolerance to salt and osmotic stress.

\section{ROS Accumulation and Scavenging Analysis}

$\mathrm{DAB}$ and NBT staining were performed to study the accumulation of $\mathrm{H}_{2} \mathrm{O}_{2}$ and $\mathrm{O}_{2}{ }^{-}$. Compared with the WT plants, both $\mathrm{H}_{2} \mathrm{O}_{2}$ and $\mathrm{O}_{2}{ }^{-}$were reduced greatly in the leaves of the two $\mathrm{OE}$ lines under salt and osmotic stress conditions (Figures 4A,B). Conversely, $\mathrm{H}_{2} \mathrm{O}_{2}$ and $\mathrm{O}_{2}{ }^{-}$in the $\mathrm{KO} 4$ and KO 5 individual plants were substantially higher than that in the WT plants (Figures 4A,B). We further determined $\mathrm{H}_{2} \mathrm{O}_{2}$ level, and the results showed that $\mathrm{H}_{2} \mathrm{O}_{2}$ content was highest in $\mathrm{KO} 4$ and KO 5 plants, followed by in WT plants, and lowest in the two OE lines, which is consistent with DAB staining (Figure 4F). We further studied whether the altered ROS level was caused the changed antioxidant activity in the plants. The levels of two main antioxidant enzymes, SOD and peroxidase (POD), which are involved in ROS scavenging, were determined. Under normal conditions, the activities of SOD and POD were similar among the studied plants (Figures 4C,D). Under $\mathrm{NaCl}$ and mannitol 

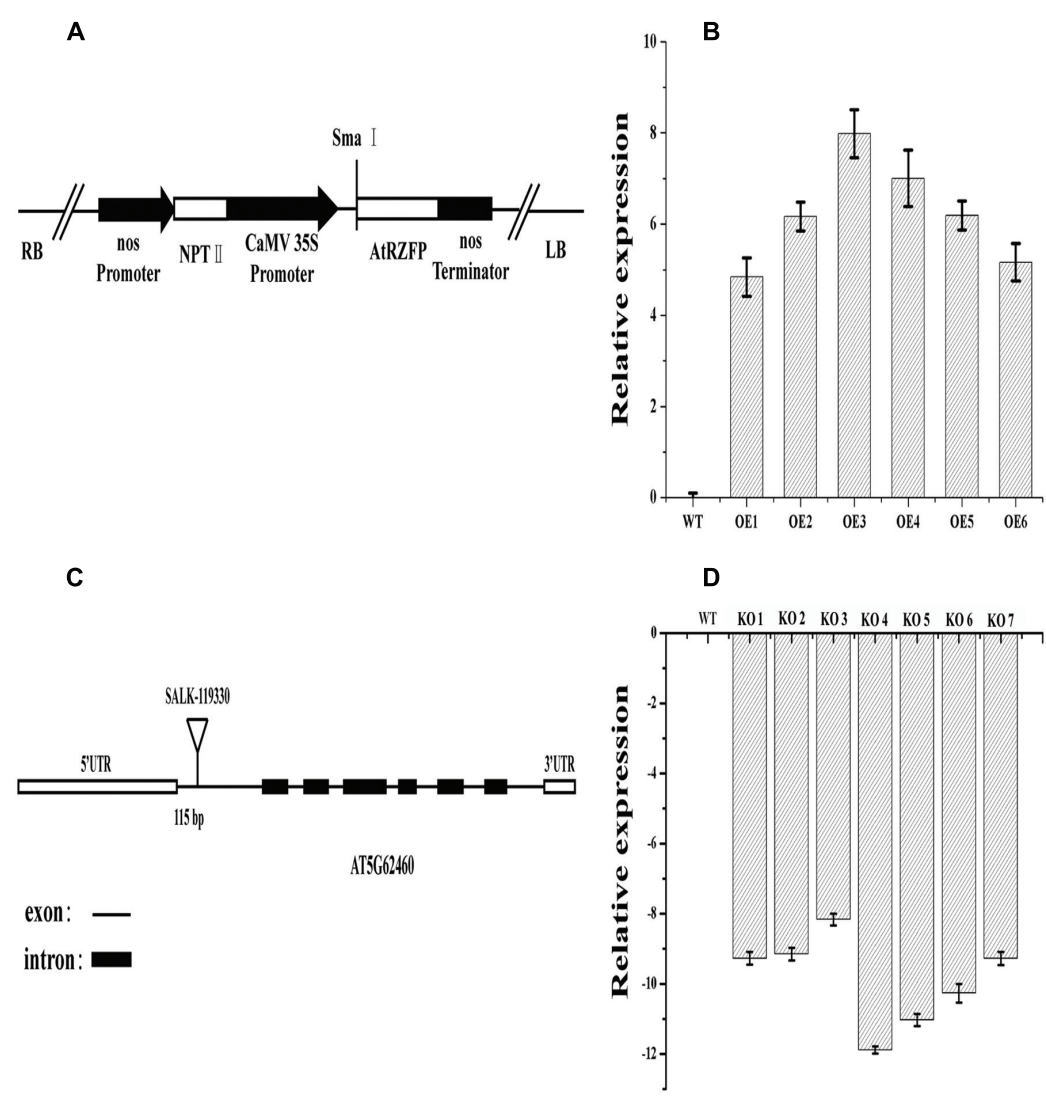

FIGURE 2 | Analysis of AtRZFP transcript levels in overexpression (OE) lines and Knockout of AtRZFP (KO) plants. (A) Diagram of the plant expression vector for the overexpression of AtRZFP. (B) The expression of AtRZFP in OE lines. (C) Diagram of the T-DNA insertion positions of T-DNA insertion positions for a single allele At5g62460. (D) The expression of AtRZFP in the KO plants (SALK_119330 individual plants). The expression level of AtRZFP in each line was normalized by the expression of AtRZFP in wild-type (WT) plants. All the expression data were log2 transformed.

treatment conditions, compared with the WT plants, both SOD and POD activities were significantly increased in the OE lines, but decreased in the KO 4 and KO 5 plants (Figures 4C,D). Correspondingly, the malondialdehyde (MDA) content analysis showed that there was no difference in MDA contents among the studied lines under normal conditions (Figure 4E). When exposed to salt or osmotic stress conditions, the MDA content in all the studied plants increased. However, the OE lines displayed lower MDA contents than the WT plants, while the KO 4 and KO 5 had the highest MDA contents among the studied lines (Figure 4E). Therefore, AtRZFP could decrease membrane lipid peroxidation generated by salt or osmotic stress to improve salt and osmotic stress.

\section{Electrolyte Leakage and MDA Content Analysis}

Electrolyte leakage was determined to monitor the level of cell death. No significant differences in electrolyte leakage rates were observed among the studied lines under normal conditions. However, under salt and osmotic stress conditions, electrolyte leakage among the $\mathrm{OE}$ lines, $\mathrm{KO} 4, \mathrm{KO} 5$, and WT plants were significantly different. Electrolyte leakages in the KO 4 and KO 5 individual plants were highest, followed by the WT, and then the OE lines, which had the lowest electrolyte leakage (Figure 5A). Consistently, Evans blue staining also confirmed that cell membrane damage was increased greatly in the KO 4 and KO 5 individual plants, but was substantially decreased in the $\mathrm{OE}$ lines (Figure 5B). These results indicated that overexpression of AtRZFP could reduce cell death to improve salt or osmotic stress tolerance.

\section{$\mathrm{Na}^{+}$and $\mathrm{K}^{+}$Content}

The contents of $\mathrm{Na}^{+}$and $\mathrm{K}^{+}$were determined in the plant leaves and roots. The results showed that under normal conditions, the $\mathrm{OE}$ lines also had a relative lower $\mathrm{Na}^{+}$level, higher $\mathrm{K}^{+}$level and higher $\mathrm{K}^{+} / \mathrm{Na}^{+}$ratio than the WT plants; conversely, the KO 4 and $\mathrm{KO} 5$ individual plants had a higher $\mathrm{Na}^{+}$content, lower $\mathrm{K}^{+}$ level and $\mathrm{K}^{+} / \mathrm{Na}^{+}$ratio than the WT plants (Figure 6). When exposed to salt stress conditions, all the studied lines showed reduced $\mathrm{K}^{+}$contents under salt treatments, but both the OE 3 and $\mathrm{OE} 4$ lines still had the highest $\mathrm{K}^{+}$content in their leaves and roots, followed by that in the WT plants, whereas both $\mathrm{KO}$ 4 and KO 5 lines had the lowest $\mathrm{K}^{+}$contents. All the studied lines showed increased $\mathrm{Na}^{+}$content under salt stress conditions. 
A

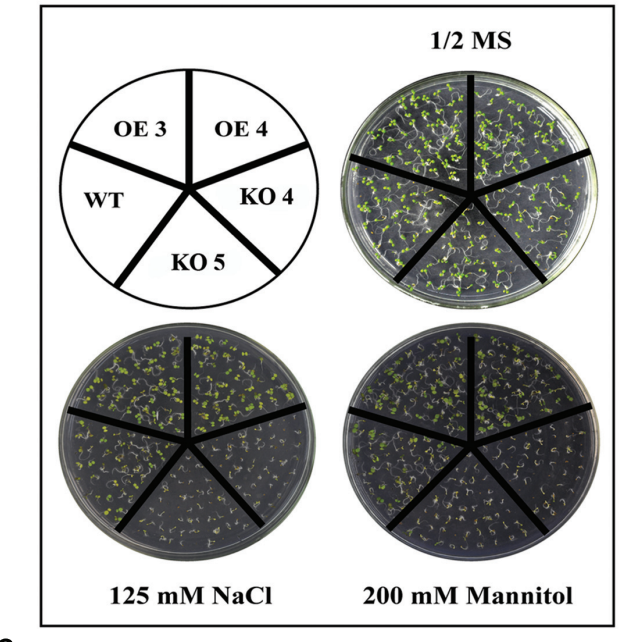

c
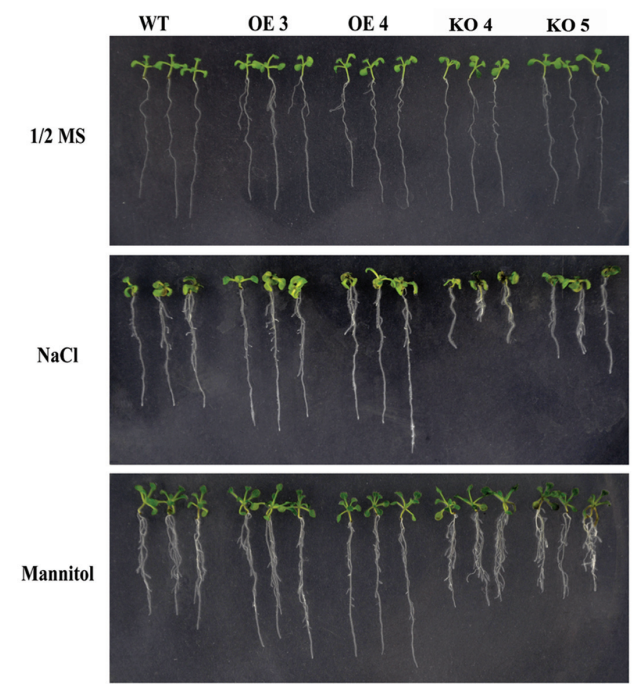

B
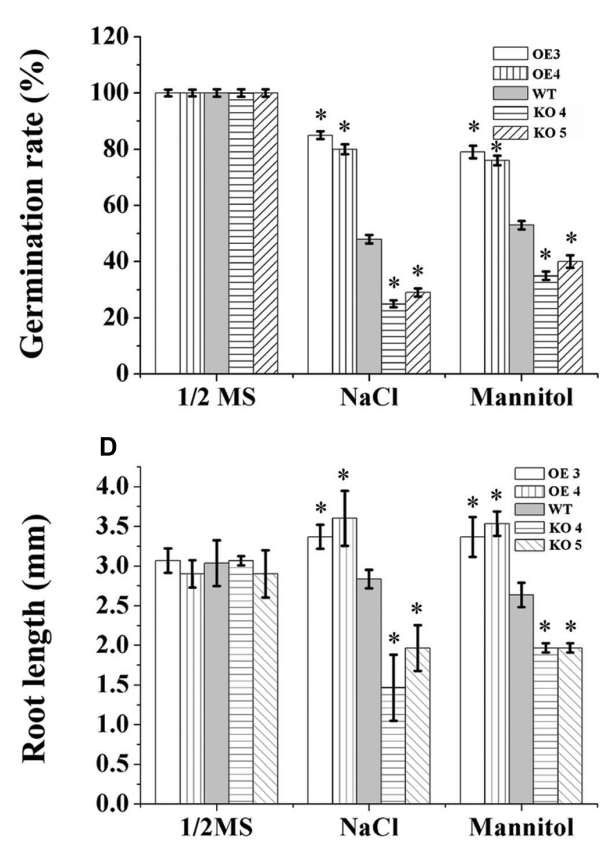

E

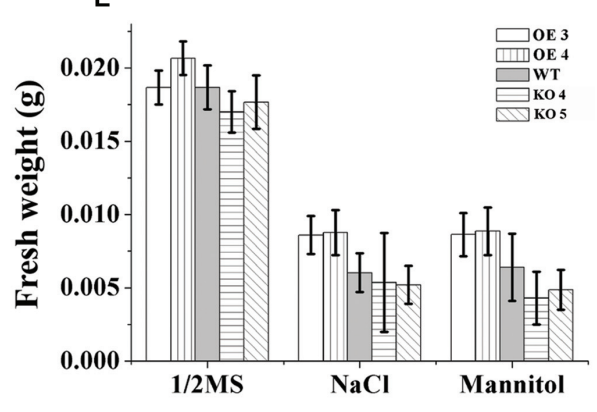

FIGURE 3 | Tolerance Analysis of AtRZFP. (A,B) Seed germination rate analysis. Seeds were grown in 1/2 or $1 / 2$ MS supplied with $150 \mathrm{mM} \mathrm{NaCl}$ or $200 \mathrm{mM}$ mannitol for 1 week, after which, the germination rates were compared. (C) Comparison of growth phenotypes. (D) Root length. (E) Analysis of fresh weight (FW). Three independent experiments were performed; bars indicate standard deviation. *Indicates significant differences between the OE lines and WT, or between WT and the SALK_119330 individual plants (KO 4 and KO 5) under the same conditions $(P<0.05)$.

However, the KO 4 and KO 5 lines accumulated significantly higher $\mathrm{Na}^{+}$contents than the WT and OE plants, and both OE lines had significant lower $\mathrm{Na}^{+}$accumulation than the WT plants (Figure 6). Additionally, both $\mathrm{OE}$ lines had the highest $\mathrm{K}^{+} / \mathrm{Na}^{+}$ ratio, followed by the WT, with the two SALK_119330 individual plants (KO 4 and $\mathrm{KO} 5$ ) having the lowest $\mathrm{K}^{+} / \mathrm{Na}^{+}$ratio under salt stress conditions.

\section{$\mathrm{Na}^{+}$Distribution Assay using CoroNa-Green Staining}

We further studied the accumulation and distribution of $\mathrm{Na}^{+}$in roots at different lines using CoroNa-Green staining. The results showed that the OE lines had relatively lower $\mathrm{Na}^{+}$contents than the WT and KO 4 and KO 5 plants under normal conditions. When exposed to salt stress, the KO 4 and KO 5 individual plants showed obviously higher $\mathrm{Na}^{+}$accumulation than the WT plants, but the OE lines showed obviously lower $\mathrm{Na}^{+}$accumulation than the WT plants (Figure 6G). These results were consistent with the measurement of $\mathrm{Na}^{+}$using the flame photometer (Figure 6), indicating that AtRZFP could regulate the accumulation of $\mathrm{Na}^{+}$ in plants in response to salt stress.

\section{Expression of AtRZFP Decreased Stomatal Apertures to Reduce Water Loss}

To study whether the conservation of water capability is altered by the changed expression of AtRZFP, water loss rates were calculated. Compared with the WT plants, the water loss rates of the OE lines were decreased significantly; however, the water 


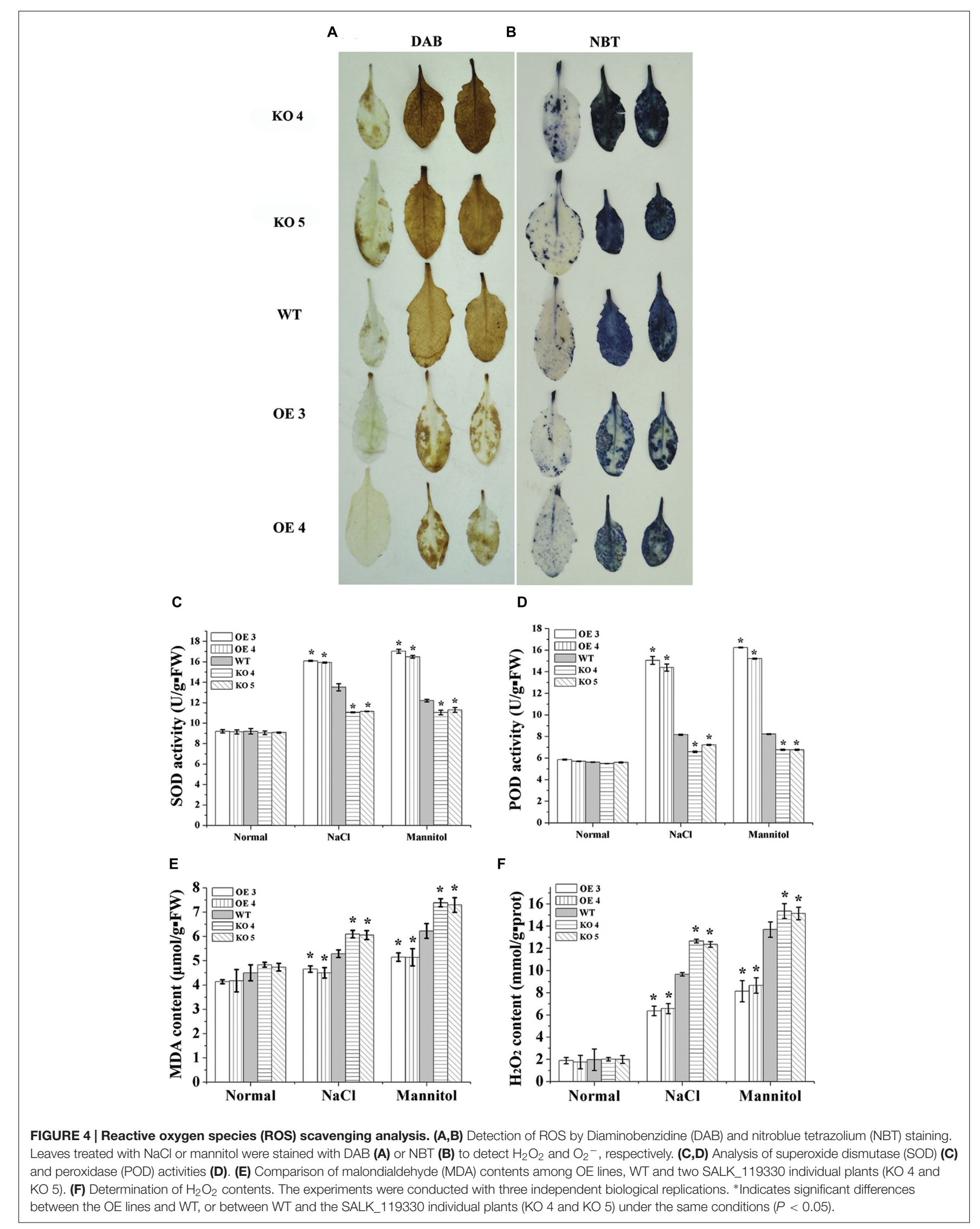



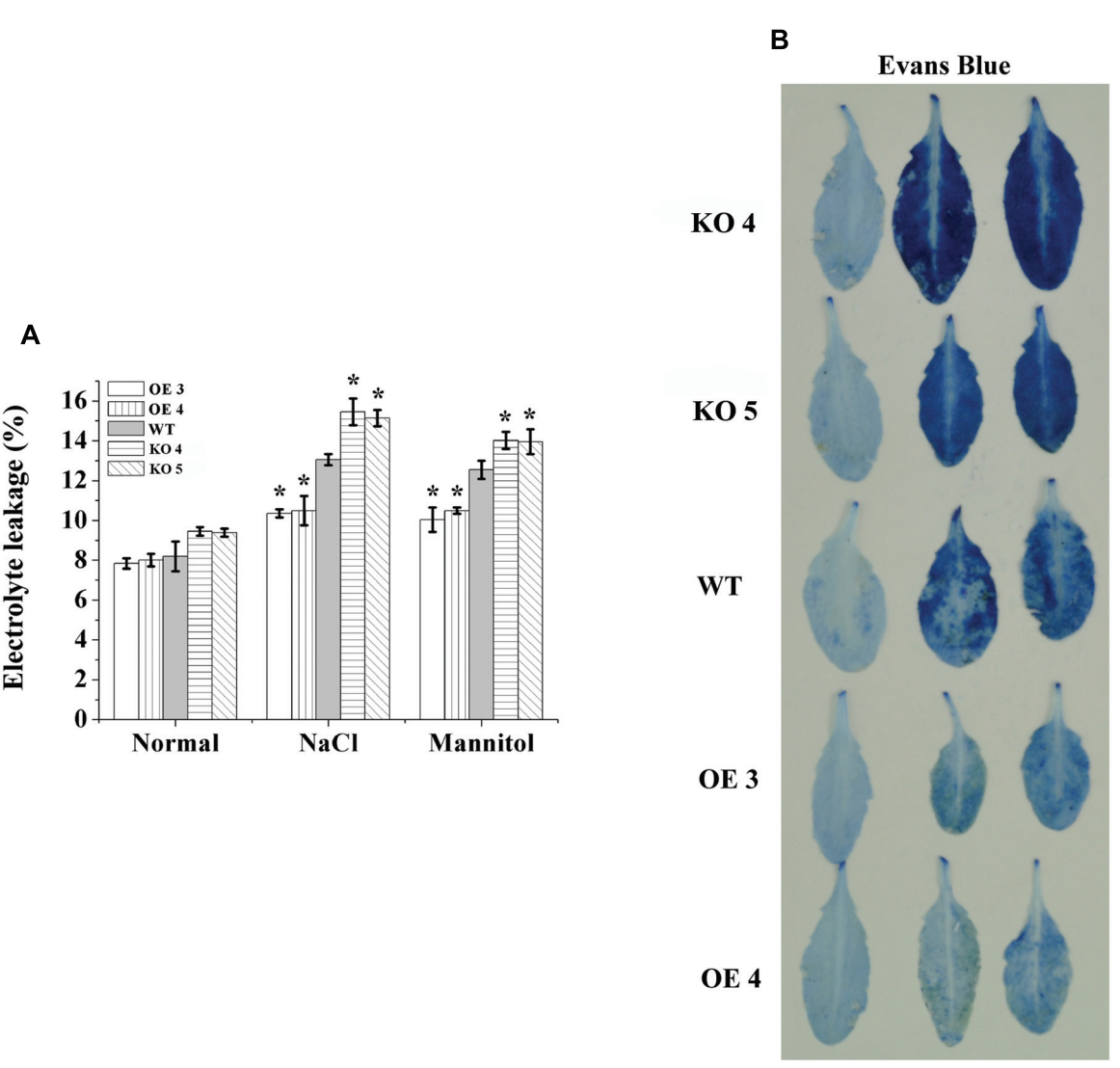

FIGURE 5 | Electrolyte leakage analysis. (A) Comparison of electrolyte leakage rates. (B) Evans blue staining assay. Three independent experiments were averaged; bars indicate standard deviation. *Indicates significant differences between the OE lines and WT, or between WT and the SALK_119330 individual plants (KO 4 and $\mathrm{KO} 5$ ) under the same conditions $(P<0.05)$.

loss rates of the KO 4 and KO 5 individual plants were increased greatly (Figure 7A).

To investigate whether the reduced water loss rate was caused by changes to stomatal apertures, we measured the stomatal apertures in leaves of the studied lines. Under normal conditions, the stomatal apertures of the studied lines were similar (Figures $\mathbf{7 B}, \mathbf{C}$ ). However, under salt or osmotic stress conditions, compared with the WT plants, both OE lines showed the smallest stomatal apertures (width/length), and the $\mathrm{KO}$ 4 and $\mathrm{KO} 5$ individual plants showed the highest stomatal apertures (Figures $\mathbf{7 B}, \mathbf{C}$ ), indicating that AtRZFP is involved in modulating the closure and opening of stomatal apertures in response to salt and osmotic stress conditions.

\section{Proline and Soluble Sugar Content Analysis}

There was no difference in the proline contents among the OE lines, $\mathrm{KO} 4$, KO 5 and WT under normal growth conditions. After salt or mannitol treatments, the WT and OE lines showed increased proline levels, while the proline levels in the KO 4 and KO 5 individual plants did not change. Additionally, in the OE lines, the proline contents increased significantly compared with the WT plants, and the proline contents in the KO 4 and KO 5 plants were lower than in the WT lines under salt and osmotic stress conditions (Figure 8).

Soluble sugar levels in different lines were quite similar under normal conditions. When exposed to salt and osmotic stress, the soluble sugar levels increased in all studied lines; however, the soluble sugar levels were increased highly in the OE lines compared with the WT and KO 4 and KO 5 plants, and both OE lines had the highest proline levels among all the lines. The KO 4 and KO 5 plants had the lowest proline levels compared with the OE and WT lines under salt and osmotic stress conditions (Figure 8).

\section{DISCUSSION}

AtRZFP is a RING/FYVE/PHD zinc finger-containing protein, which is suggested to have the function of zinc ion binding, according to the annotation in $\mathrm{TAIR}^{2}$. However, to date, the function of AtRZFP had received little attention. Additionally, the functions of AtRZFP or its homologs in response to abiotic stress were unknown. In the present study, we showed that

\footnotetext{
${ }^{2}$ http://www.arabidopsis.org/
} 

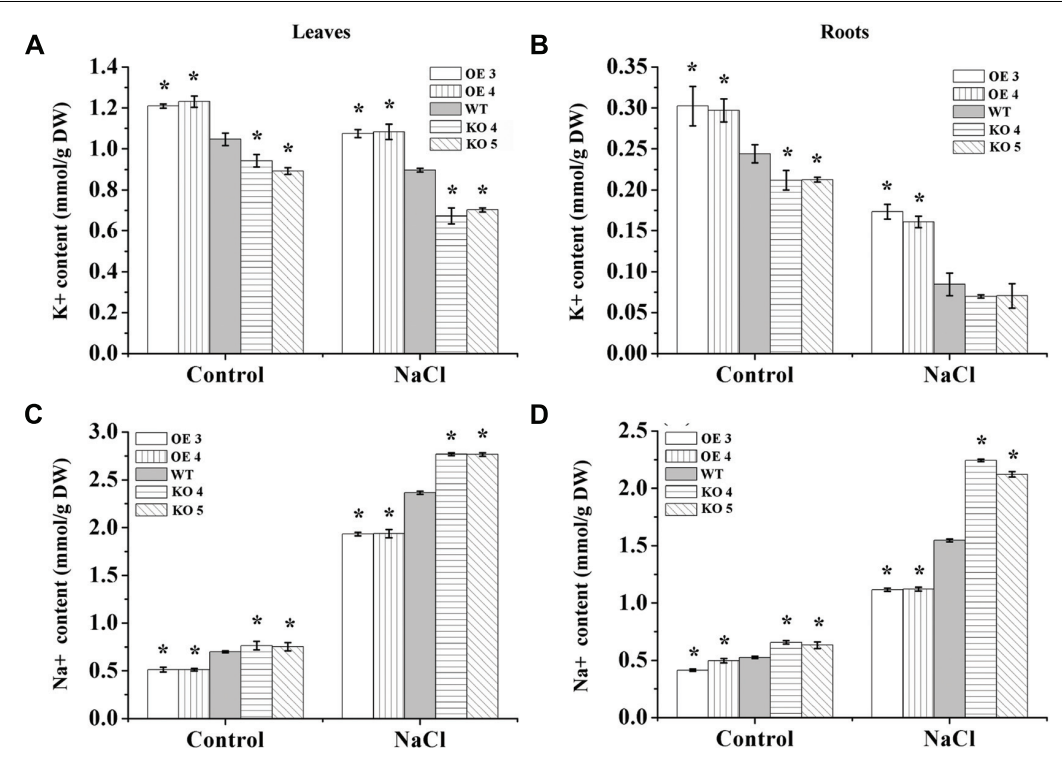

E

F
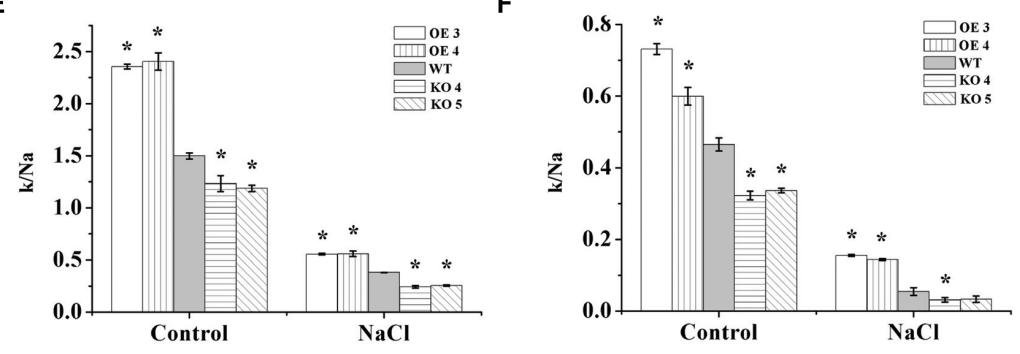

G

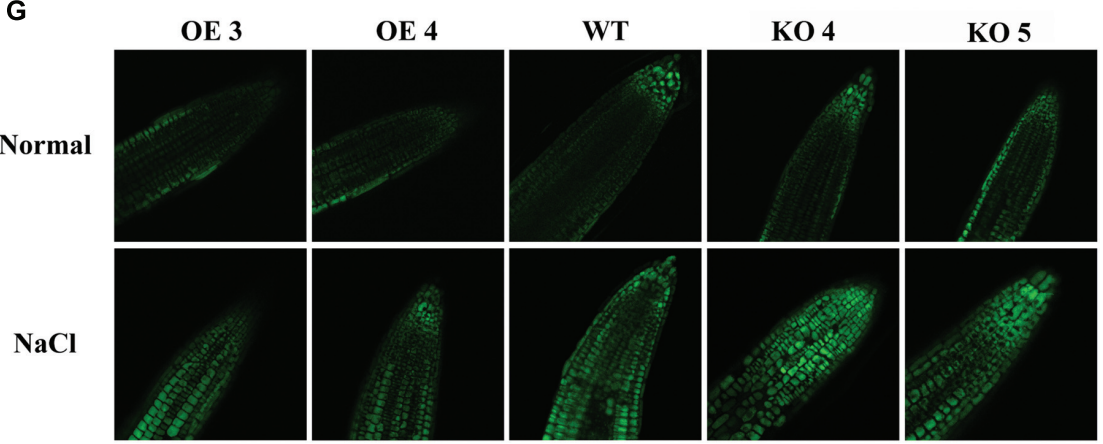

FIGURE 6 | Analyses of $\mathbf{N a}^{+}$and $\mathbf{K}^{+}$contents. One week-old Arabidopsis seedlings grown in a greenhouse were treated with $150 \mathrm{mM} \mathrm{NaCl}$ for 7 days, and plants grown under normal conditions were used as controls. (A,B) $\mathrm{K}^{+}$content in leaves (A) and roots (B). (C,D) Na ${ }^{+}$content in leaves (C) and roots (D). (E,F) $\mathrm{K}^{+} / \mathrm{Na}^{+}$ratio in leaves $(\mathbf{E})$ and roots $(\mathbf{F})$; *indicates significant differences between the OE lines and WT, or between WT and the SALK_119330 individual plants (KO 4 and $\mathrm{KO} 5)$ under the same conditions $(P<0.05)$. (G) CoroNa-Green staining analysis of $\mathrm{Na}^{+}$accumulation under normal or salt stress conditions. $\mathrm{Na}^{+}$was stained by CoroNa-Green and shown as green fluorescence; the root tips of each line were observed. The experiments were conducted with three independent biological replications.

AtRZFP regulates abiotic stress tolerance positively (Figure 3). However, AtRZFP plays a role in abiotic stress tolerance, and we found that a series of physiological responses related to abiotic stress tolerance (particularly to salt and osmotic stress) were changed when the expression of AtRZFP was altered.

$\mathrm{Na}^{+}$is one of the most toxic ions, and high $\mathrm{Na}^{+}$inhibits enzyme activity and disrupts $\mathrm{K}^{+}$uptake, leading to $\mathrm{K}^{+}$ deficiency in the cytoplasm (Maathuis, 2009; Ma et al., 2011). Therefore, reduced $\mathrm{Na}^{+}$accumulation is quite important for stress tolerance. In the present study, both CoroNa-Green staining and $\mathrm{Na}^{+}$measurement showed that the expression level of AtRZFP is negatively correlated with $\mathrm{Na}^{+}$accumulation (Figure 6), indicating that AtRZFP plays a role in preventing $\mathrm{Na}^{+}$accumulation under salt stress conditions. Sufficient $\mathrm{K}^{+}$ is important for cellular osmotic adjustment and the activities of many enzymes (Zhu, 2003), and there is a strong positive correlation between salt tolerance and the ability to retain $\mathrm{K}^{+}$in plants under salinity stress (Chen et al., 2005, 2007; 


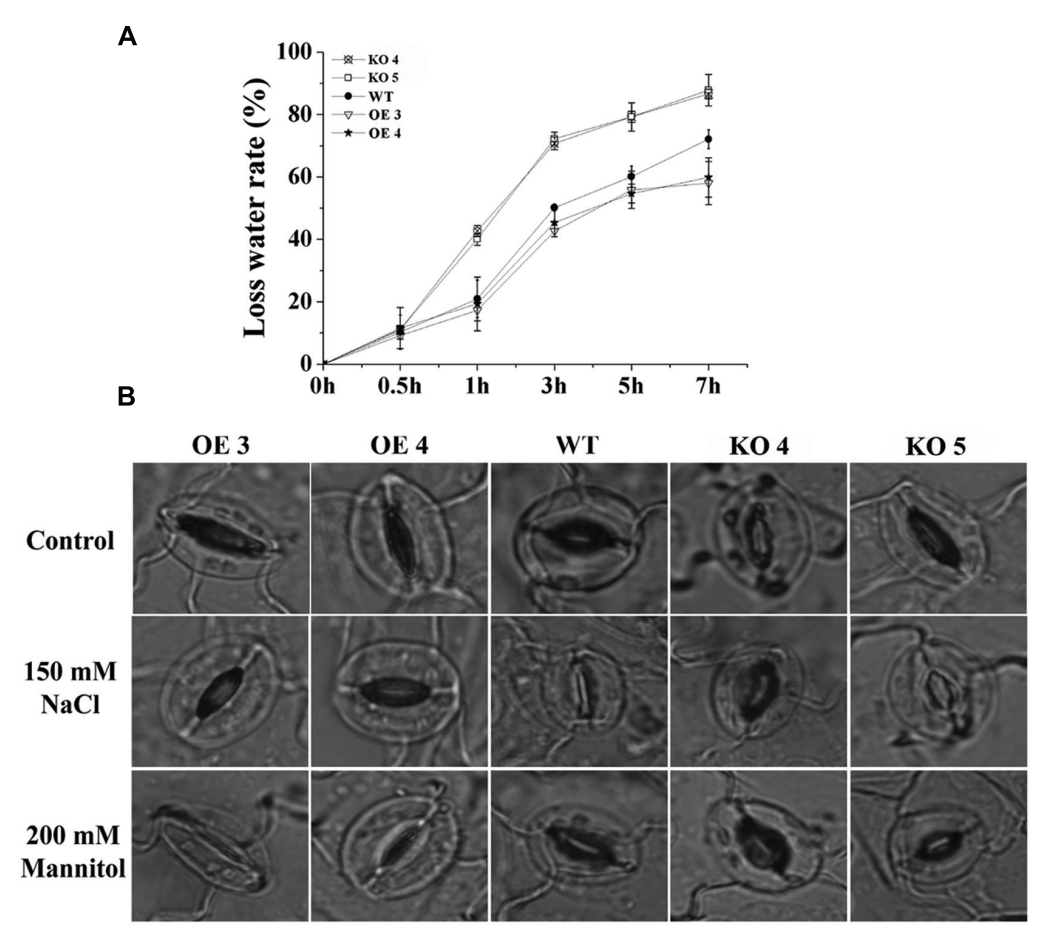

C

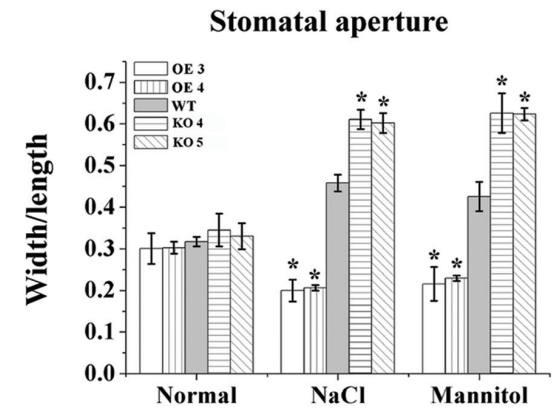

FIGURE 7 | Analysis of water loss rates and the stomatal aperture. (A) Water loss rates. (B) The stomatal aperture in each line under normal, salt or mannitol stress conditions. (C) Calculation of the width/ length ratio of the stomatal aperture in each line. The experiments were conducted with three independent biological replications. *Indicates significant differences between the OE lines and WT, or between WT and the SALK_119330 individual plants (KO 4 and KO 5) under the same conditions $(P<0.05)$.
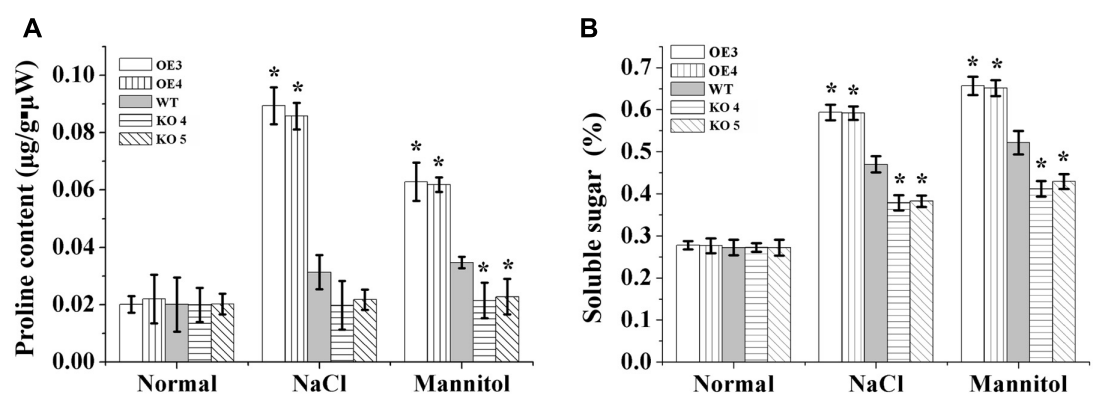

FIGURE 8 | Analysis of proline biosynthesis and soluble sugar mediated by AtRZFP contents under $\mathrm{NaCl}$ and mannitol treatment conditions.

(A) Analysis of the proline level in OE lines, WT and KO 4 and KO 5 plants. (B) Analysis of soluble sugar contents in OE lines, WT and KO 4 and KO 5 plants. Three independent biological replications were performed; bars indicate the standard deviation. *Indicates significant differences between the OE lines and WT, or between WT and the SALK_119330 individual plants (KO 4 and KO 5) under the same conditions $(P<0.05)$. 
Cuin et al., 2008). Our results showed that the transcription level of AtRZFP was positively correlated with $\mathrm{K}^{+}$contents (Figure 6), suggesting that AtRZFP plays a role in preventing loss of $\mathrm{K}^{+}$ under salt stress. Therefore, expression of AtRZFP could reduce $\mathrm{Na}^{+}$accumulation, and prevent $\mathrm{K}^{+}$loss. The reduced $\mathrm{Na}^{+}$ accumulation might contribute to avoiding toxic ion damage. The relatively high level of $\mathrm{K}^{+}$might play a role in maintaining cellular osmotic adjustment and enzyme activities, leading to significantly improved salt stress tolerance.

Stomatal regulation is a key process that links gas exchange and transpiration (Brodribb and Holbrook, 2003), and partial or complete closure of stomata could help plants to maintain a favorable water balance (Tombesi et al., 2015). Under abiotic stress conditions, reduction of the stomatal aperture is a common strategy employed by plants to avoid further water loss, and is important for plant survival (Moldau et al., 2011). Our study showed that overexpression of AtRZFP reduced the stomatal aperture, whereas knockout of AtRZFP increased the stomatal aperture (Figures $7 \mathbf{B}, \mathbf{C}$ ), suggesting that AtRZFP could affect the closure/opening of the stomatal aperture to maintain a favorable water balance, thereby enhancing salt and osmotic stress tolerance.

Some ZFPs mediate stress tolerance to adjust osmotic potential (Luo et al., 2012a; Wang et al., 2016). In the present study, the transcription of AtRZFP correlated positively with soluble sugars and proline levels, suggesting that AtRZFP might modulate the biosynthesis of soluble sugars and proline positively (Figure 8). Sugars and proline are both important compatible osmolytes in the adjustment of plants' osmotic potential (Zhang X. et al., 2011); therefore, these results suggested that AtRZFP could adjust the osmotic pressure to control stress tolerance.

Some ZFP subfamilies play important roles in regulating ROS scavenging capabilities when exposed to abiotic stress (Davletova et al., 2005; Baek et al., 2015). Consistent with these studies, our results showed that the expression of AtRZFP decreased ROS accumulation and enhanced SOD and POD activities (Figure 4), suggesting that AtRZFP might improve ROS scavenging by increasing the activities of SOD and POD, leading to reduced ROS accumulation.

In the present study, to determine the function of AtRZFP, we used AtRZFP mutant line SALK_119330 for loss-offunction analysis, which is only one knock-down allele mutation

\section{REFERENCES}

Baek, D., Cha, J. Y., Kang, S., Park, B., Lee, H. J., Hong, H., et al. (2015). The Arabidopsis a zinc finger domain protein ARS1 is essential for seed germination and ROS homeostasis in response to ABA and oxidative stress. Front.Plant Sci. 6:963. doi: 10.3389/fpls.2015.00963

Bates, L. S., Waldren, R. P., and Teare, I. D. (1973). Rapid determination of free proline for water-stress studies. Plant Soil 39, 205-207. doi: 10.1016/j.dental.2010.07.006

Bogamuwa, S., and Jang, J. C. (2016). Plant tandem CCCH Zinc finger proteins interact with $\mathrm{ABA}$, drought, and stress response regulators in processing-bodies and stress granules. PLoS ONE 11:e0151574. doi: 10.1371/journal.pone.0151574

Brodribb, T. J., and Holbrook, N. M. (2003). Stomatal closure during leaf dehydration, correlation with other leaf physiological traits. Plant Physiol. 132, 2166-2173. doi: 10.1104/pp.103.023879 plant. However, there was a shortcoming in study that we had not performed the complementary experiment. Namely, SALK_119330 plants were transformed with AtRZFP that is under the control of its native promoter. This complementary study will demonstrate that the observed phenotypes are indeed due to the mutation in AtRZFP, and need to be conducted in the future study.

\section{CONCLUSION}

We showed that AtRZFP is involved in salt and osmotic stress tolerance. When exposed to abiotic stress, AtRZFP is induced by salt or osmotic stress. The expression of AtRZFP inhibited $\mathrm{Na}^{+}$accumulation and reduced $\mathrm{K}^{+}$loss. AtRZFP also controls the stomatal aperture to maintain the water loss balance. Additionally, similar to other studied ZFPs, RING/FYVE/PHD AtRZFP also enhances ROS scavenging and regulates the osmotic potential positively to confer abiotic stress tolerance.

\section{AUTHOR CONTRIBUTIONS}

YW designed research and wrote the paper. DZ performed research. DZ and HX analyzed data. WZ and HL contributed to genes expression analysis. YZ and XS cloned the gene. YW revised the manuscript. All the authors reviewed the manuscript. All authors have no competing financial interest.

\section{ACKNOWLEDGMENTS}

This work was supported by the project of the culture of the young scientists in scientific innovation in Xinjiang Province (2013711001) and the Fundamental Research Funds for the Central Universities (2572015AA08).

\section{SUPPLEMENTARY MATERIAL}

The Supplementary Material for this article can be found online at: http://journal.frontiersin.org/article/10.3389/fpls.2016.01272

Cao, H., Huang, P., Zhang, L., Shi, Y., Sun, D., Yan, Y., et al. (2016). Characterization of 47 Cys2 -His2 zinc finger proteins required for the development and pathogenicity of the rice blast fungus Magnaporthe oryzae. New Phytol. 211, 1035-1051. doi: 10.1111/nph.13948

Chai, G., Kong, Y., Zhu, M., Yu, L., Qi, G., Tang, X., et al. (2015). Arabidopsis $\mathrm{C} 3 \mathrm{H} 14$ and $\mathrm{C} 3 \mathrm{H} 15$ have overlapping roles in the regulation of secondary wall thickening and anther development. J. Exp. Bot. 66, 2595-2609. doi: $10.1093 /$ jxb/erv060

Chen, Z., Newman, I., Zhou, M., Mendham, N., Zhang, G., and Shabala, S. (2005). Screening plants for salt tolerance by measuring $\mathrm{K}+$ flux: a case study for barley. Plant Cell Environ. 28, 1230-1246. doi: 10.1111/j.1365-3040.2005. 01364.x

Chen, Z., Pottosin, I. I., Cuin, T. A., Fuglsang, A. T., Tester, M., Jha, D., et al. (2007). Root plasma membrane transporters controlling $\mathrm{K}+/ \mathrm{Na}+$ homeostasis in saltstressed barley. Plant Physiol. 145, 1714-1725. doi: 10.1104/pp.107.110262 
Cheuk, A., and Houde, M. (2016). Genome wide identification of C1-2i zinc finger proteins and their response to abiotic stress in hexaploid wheat. Mol. Genet. Genomics 291, 873-890. doi: 10.1007/s00438-015-1152-1

Cuin, T. A., Betts, S. A., Chalmandrier, R., and Shabala, S. (2008). A root's ability to retain $\mathrm{K}+$ correlates with salt tolerance in wheat. J. Exp. Bot. 59, 2697-2706. doi: $10.1093 /$ jxb/ern 128

Davletova, S., Schlauch, K., Coutu, J., and Mittler, R. (2005). The zinc-finger protein Zat12 plays a central role in reactive oxygen and abiotic stress signaling in Arabidopsis. Plant Physiol. 139, 847-856. doi: 10.1104/pp.105. 068254

Dhindsa, R. S., Plumb-Dhindsa, P., and Thorpe, T. A. (1981). Leaf senescence: correlated with increased levels of membrane permeability and lipid peroxidation, and decreased levels of superoxide dismutase and catalase. J. Exp. Bot. 32, 93-101. doi: 10.1093/jxb/32.1.93

Dong, H., and Chin, B. (2000). Chilling stress induced changes of antioxidant enzymes in the leaves of cucumber: in gel enzymes activity as says. Plant Sci. 159, 75-85. doi: 10.1016/S0168-9452(00)00326-5

D’Orso, F., De Leonardis, A. M., Salvi, S., Gadaleta, A., Ruberti, I., Cattivelli, L., et al. (2015). Conservation of AtTZF1, AtTZF2, and AtTZF3 homolog gene regulation by salt stress in evolutionarily distant plant species. Front. Plant Sci. 6:394. doi: 10.3389/fpls.2015.00394

Fan, W., Lou, H. Q., Gong, Y. L., Liu, M. Y., Cao, M. J., Liu, Y., et al. (2015). Characterization of an inducible $\mathrm{C} 2 \mathrm{H} 2$-type zinc finger transcription factor VuSTOP1 in rice bean (Vigna umbellata) reveals differential regulation between low pH and aluminum tolerance mechanisms. New Phytol. 208, 456-468. doi: 10.1111/nph.13456

Fryer, M. J., Oxborough, K., Mullineaux, P. M., and Baker, N. R. (2002). Imaging of photo-oxidative stress responses in leaves. J. Exp. Bot. 53, 1249-1254. doi: 10.1093/jexbot/53.372.1249

Gao, H., Song, A., Zhu, X., Chen, F., Jiang, J., Chen, Y., et al. (2012). The heterologous expression in Arabidopsis of a chrysanthemum Cys2/His2 zinc finger protein gene confers salinity and drought tolerance. Planta 235, 979-993. doi: 10.1007/s00425-011-1558-x

Gupta, S. K., Rai, A. K., Kanwar, S. S., and Sharma, T. R. (2012). Comparative analysis of zinc finger proteins involved in plant disease resistance. PLoS ONE 7:e42578. doi: 10.1371/journal.pone.0042578

Han, Y., Jiang, J., Liu, H., Ma, Q., Xu, W., Xu, Y., et al. (2005). Overexpression of OsSIN, encoding a novel small protein, causes short internodes in Oryza sativa. Plant Sci. 169, 487-495. doi: 10.1016/j.plantsci.2005.03.029

Hilder, V. A., Gatehouse, A. M., Sheerman, S. E., Barker, R. F., and Boulter, D. (1987). A novel mechanism of insect resistance engineered into tobacco. Nature 330, 160-163. doi: 10.1038/330160a0

Jefferson, R. A., Kavanagh, T. A., and Bevan, M. W. (1987). GUS fusions:betaglucuronidase as a sensitive and versatile gene fusion markerin higher plants. EMBO J. 6, 3901-3907.

Kadir, F. A., Kassim, N. M., Abdulla, M. A., and Yehye, W. A. (2013). PASSpredicted Vitex negundo activity: antioxidant and antiproliferative properties on human hepatoma cells-an in vitro study. BMC Complement Altern. Med. 13:343. doi: 10.1186/1472-6882-13-343

Kim, G. D., Cho, Y. H., and Yoo, S. D. (2015). Regulatory functions of evolutionarily conserved AN1/A20-like Zinc finger family proteins in Arabidopsis stress responses under high temperature. Biochem. Biophys. Res. Commun. 457, 213-220. doi: 10.1016/j.bbrc.2014.12.090

Le, C. T. T., Brumbarova, T., Ivanov, R., Stoof, C., Weber, E., Mohrbacher, J., et al. (2016). ZINC FINGER OF ARABIDOPSIS THALIANA12 (ZAT12) Interacts with FER-LIKE IRON DEFICIENCY-INDUCED TRANSCRIPTION FACTOR (FIT) Linking Iron Deficiency and Oxidative Stress Responses. Plant Physiol. 170, 540-557. doi: 10.1104/pp.15.01589

Li, W. T., Chen, W. L., Yang, C., Wang, J., Yang, L., He, M., et al. (2014). Identification and network construction of zinc finger protein (ZFP) genes involved in the rice-Magnaporthe oryzae interaction. Plant Omics 7, 540-548.

Li, X. J., Guo, C. J., Lu, W. J., Duan, W. W., Miao, Z., Gu, J. T., et al. (2014). Expression pattern analysis of zinc finger protein genes in wheat (Triticum aestivum L.) under phosphorus deprivation. J. Integr. Agric. 13, 1621-1633. doi: 10.1016/S2095-3119(13)60739-X

Li, Y., Yue, X., Que, Y., Yan, X., Ma, Z., Talbot, N. J., et al. (2014). Characterisation of four LIM protein-encoding genes involved in infection-related development and pathogenicity by the rice blast fungus Magnaporthe oryzae. PLoS ONE 9:e88246. doi: 10.1371/journal.pone.0088246

Liu, Q., Wang, Z., Xu, X., Zhang, H., and Li, C. (2015). Genome-wide analysis of $\mathrm{C} 2 \mathrm{H} 2$ Zinc-finger family transcription factors and their responses to abiotic stresses in poplar (Populus trichocarpa). PLoS ONE 10:e0134753. doi: 10.1371/journal.pone.0134753

Liu, X. M., Nguyen, X. C., Kim, K. E., Han, H. J., Yoo, J., Lee, K., et al. (2013). Phosphorylation of the zinc finger transcriptional regulator ZAT6 by MPK6 regulates Arabidopsis seed germination under salt and osmotic stress. Biochem. Biophys. Res. Commun. 430, 1054-1059. doi: 10.1016/j.bbrc.2012. 12.039

Liu, Y., Xu, Y., Xiao, J., Ma, Q., Li, D., Xue, Z., et al. (2011). OsDOG, a gibberellin-induced A20/AN1 zinc-finger protein, negatively regulates gibberellin-mediated cell elongation in rice. J. Plant Physiol. 168, 1098-1105. doi: 10.1016/j.jplph.2010.12.013

Luo, X., Bai, X., Zhu, D., Li, Y., Ji, W., Cai, H., et al. (2012a). GsZFP1, a new Cys2/His2-type zinc-finger protein, is a positive regulator of plant tolerance to cold and drought stress. Planta 235, 1141-1155. doi: 10.1007/s00425-0111563-0

Luo, X., Cui, N., Zhu, Y., Cao, L., Zhai, H., Cai, H., et al. (2012b). Over-expression of GsZFP1, an ABA-responsive C2H2-type zinc finger protein lacking a QALGGH motif, reduces ABA sensitivity and decreases stomata size. J. Plant Physiol. 169, 1192-1202. doi: 10.1016/j.jplph.2012. 03.019

Ma, L., Zhang, H., Sun, L., Jiao, Y., Zhang, G., Miao, C., et al. (2011). NADPH oxidase AtrbohD and AtrbohF function in ROS-dependent regulation of $\mathrm{Na}+/ \mathrm{K}+$ homeostasis in Arabidopsis under salt stress. J. Exp. Bot. 63, 305-317. doi: $10.1093 / \mathrm{jxb} / \mathrm{err} 280$

Maathuis, F. J. (2009). Physiological functions of mineral macronutrients. Curr. Opin. Plant Biol. 12, 250-258. doi: 10.1016/j.pbi.2009.04.003

Miller, G., Shulaev, V., and Mittler, R. (2008). Reactive oxygen signaling and abiotic stress. Physiol. Plant 133, 481-489. doi: 10.1111/j.1399-3054.2008. 01090.x

Moldau, H., Vahisalu, T., and Kollist, H. (2011). Rapid stomatal closure triggered by a short ozone pulse is followed by reopening to overshooting values. Plant Signal. Behav. 6, 311-313. doi: 10.4161/psb.6.2.15044

Oh, D. H., Lee, S. Y., Bressan, R. A., Yun, D. J., and Bohnert, H. J. (2010). Intracellular consequences of SOS1 deficiency during salt stress. J. Exp. Bot. 61, 1205-1213. doi: 10.1093/jxb/erp391

Pei, Z. M., Kuchitsu, K., Ward, J. M., Schwarz, M., and Schroeder, J. I. (1997). Differential abscisic acid regulation of guard cell slow anion channels in Arabidopsis wild-type and abil and abi2 mutants. Plant Cell 9, 409-423. doi: $10.2307 / 3870491$

Schumann, U., Prestele, J., O'Geen, H., Brueggeman, R., Wanner, G., and Gietl, C. (2007). Requirement of the C3HC4 zinc RING finger of the Arabidopsis PEX10 for photorespiration and leaf peroxisome contact with chloroplasts. Proc. Natl. Acad. Sci. U.S.A. 104, 1069-1074. doi: 10.1073/pnas.061 0402104

Sendon, P. M., Oo, M. M., Park, J. B., Lee, B. H., Kim, J. H., Seo, H. S., et al. (2014). Activation of $\mathrm{C} 2 \mathrm{H} 2$-type zinc finger genes induces dwarfism in Arabidopsis thaliana. J. Korean Soc. Appl. Biol. Chem. 57, 35-41. doi: 10.1007/s13765-0134276-1

Seok, H. Y., Woo, D. H., Park, H. Y., Lee, S. Y., Tran, H. T., Lee, E. H., et al. (2016). AtC3H17, a non-tandem CCCH zinc finger protein, functions as a nuclear transcriptional activator and has pleiotropic effects on vegetative development, flowering and seed development in Arabidopsis. Plant Cell Physiol. 57, 603-615. doi: 10.1093/pcp/pcw013

Sreenivasulu, N., Grimm, B., and Wobus, U. (2000). Differential response of antioxidant compounds to salinity stress in salt tolerant and salt sensitive seedling of foxital millet(Setaria italica). Physiol. Plant 109, 435-442. doi: 10.1034/j.1399-3054.2000.100410.x

Sun, S. J., Guo, S. Q., Yang, X., Bao, Y. M., Tang, H. J., Sun, H., et al. (2010). Functional analysis of a novel Cys2/His2-type zinc finger protein involved in salt tolerance in rice. J. Exp. Bot. 61, 2807-2818. doi: 10.1093/jxb/ erq120

Thordal-Christensen, H., Zhang, Z., Wei, Y., and Collinge, D. B. (1997). Subcellular localization of $\mathrm{H} 2 \mathrm{O} 2$ in plants. $\mathrm{H} 2 \mathrm{O} 2$ accumulation in papillae and 
hypersensitive response during the barley-powdery mildew interaction. Plant J. 11, 1187-1194.

Tombesi, S., Nardini, A., Frioni, T., Soccolini, M., Zadra, C., Farinelli, D., et al. (2015). Stomatal closure is induced by hydraulic signals and maintained by ABA in drought-stressed grapevine. Sci. Rep. 5:12449. doi: 10.1038/srep12449

Wang, F., Tong, W., Zhu, H., Kong, W., Peng, R., Liu, Q., et al. (2016). A novel Cys2/His2 zinc finger protein gene from sweetpotato, IbZFP1, is involved in salt and drought tolerance in transgenic Arabidopsis. Planta 243, 783-797. doi: 10.1007/s00425-015-2443-9

Weng, L., Zhao, F., Li, R., Xu, C., Chen, K., and Xiao, H. (2015). The zinc finger transcription factor SlZFP2 negatively regulates abscisic acid biosynthesis and fruit ripening in tomato. Plant Physiol. 167, 931-949. doi: 10.1104/pp.114.255174

Yang, Y., Ma, C., Xu, Y., Wei, Q., Imtiaz, M., Lan, H., et al. (2014). A zinc finger protein regulates flowering time and abiotic stress tolerance in chrysanthemum by modulating gibberellin biosynthesis. Plant Cell 26, 2038 2054. doi: $10.1105 /$ tpc. 114.124867

Zhang, D., Tong, J., Xu, Z., Wei, P., Xu, L., Wan, Q., et al. (2016). Soybean C2H2type zinc finger protein GmZFP3 with conserved QALGGH motif negatively regulates drought responses in transgenic Arabidopsis. Front. Plant Sci. 7:325. doi: $10.3389 /$ fpls.2016.00325

Zhang, H., Liu, Y., Wen, F., Yao, D., Wang, L., Guo, J., et al. (2014). A novel rice $\mathrm{C} 2 \mathrm{H} 2$-type zinc finger protein, ZFP36, is a key player involved in abscisic acidinduced antioxidant defence and oxidative stress tolerance in rice. J. Exp. Bot. 65, 5795-5809.
Zhang, L., Xiao, S., Li, W., Feng, W., Li, J., Wu, Z., et al. (2011). Overexpression of a Harpin-encoding gene hrf1 in rice enhances drought tolerance. J. Exp. Bot. 62, 4229-4238. doi: 10.1093/jxb/err131

Zhang, X., Wang, L., Meng, H., Wen, H., Fan, Y., and Zhao, J. (2011). Maize ABP9 enhances tolerance to multiple stresses in transgenic Arabidopsis by modulating ABA signaling and cellular levels of reactive oxygen species. Plant Mol. Biol. 75, 365-378. doi: 10.1007/s11103-011-9732-X

Zhang, Y., Lan, H., Shao, Q., Wang, R., Chen, H., Tang, H., et al. (2016). An A20/AN1-type zinc finger protein modulates gibberellins and abscisic acid contents and increases sensitivity to abiotic stress in rice (Oryza sativa). J. Exp. Bot. 67, 315-326. doi: 10.1093/jxb/erv464

Zhu, J. K. (2003). Regulation of ion homeostasis under salt stress. Curr. Opin. Plant Biol. 6, 441-445. doi: 10.1016/S1369-5266(03)00085-2

Conflict of Interest Statement: The authors declare that the research was conducted in the absence of any commercial or financial relationships that could be construed as a potential conflict of interest.

Copyright (c) 2016 Zang, Li, Xu, Zhang, Zhang, Shi and Wang. This is an openaccess article distributed under the terms of the Creative Commons Attribution License (CC BY). The use, distribution or reproduction in other forums is permitted, provided the original author(s) or licensor are credited and that the original publication in this journal is cited, in accordance with accepted academic practice. No use, distribution or reproduction is permitted which does not comply with these terms. 